\section{Tendências temporais do consumo de frutas e hortaliças entre adultos nas capitais brasileiras e Distrito Federal, 2008-2016}

\author{
Time trends in the consumption of fruits and \\ vegetables among adults in Brazilian state \\ capitals and the Federal District, 2008-2016
}
Tendencias temporales del consumo de frutas y hortalizas entre adultos en capitales brasileñas
y Distrito Federal, 2008-2016

Luiza Eunice Sá da Silva 1

Rafael Moreira Claro 1

\section{Resumo}

Analisar a tendência temporal do consumo de frutas e hortaliças entre adultos nas capitais brasileiras e no Distrito Federal no período entre 2008 e 2016. Trata-se de um estudo de série temporal, com dados do VIGITEL de 2008 a 2016 ( $n=463.817)$. Analisou-se o percentual de consumo regular ( $\geq 5$ dias/ semana) e recomendado ( $\geq 5 x /$ dia) de frutas e hortaliças, para cada um dos anos, para o conjunto completo da população e segundo sexo, faixa de idade, nível de escolaridade e localidade. A presença de tendência linear (de aumento ou diminuição) na variação dos indicadores foi analisada por meio da regressão Prais-Winsten. Verificou-se aumento significativo $(p<0,05)$ na prevalência de consumo regular (de 33 a 35,2\%, aumento de 1,86\%/ano) e de consumo recomendado de frutas e hortaliças (de 20 a 24,4\%, aumento de 3,32\%/ano). Tendência semelhante foi identificada para o percentual da população atendendo a recomendação de consumo na maior parte dos estratos populacionais, com maior magnitude de aumento entre: homens (4\%/ano vs. 3,05\%/ano para as mulheres), adultos jovens (3,97\%/ano para aqueles com idades entre 18 e 24 anos vs. 2,3\%/ano para os que têm de 55-64 anos), aqueles com menor escolaridade (2,97\%/ano para aqueles com 0-8 anos de estudos vs. 2,76\%/ano para os com 12 anos ou mais) e residentes em regiões menos desenvolvidas (5,02\%/ano na Região Norte vs. 2,6\%/ano na Região Sudeste). Observou-se aumento do consumo de frutas e hortaliças especialmente entre os grupos com menor nível de consumo no início do período estudado. No entanto, 3 a cada 4 indivíduos permanecem consumindo menos frutas e hortaliças do que o recomendado.

Frutas; Hortaliças; Consumo de Alimentos; Doença Crônica

\author{
Correspondência \\ L. E. S. Silva \\ Universidade Federal de Minas Gerais. \\ Av. Professor Alfredo Balena 190, Belo Horizonte, MG \\ 30130-100, Brasil. \\ luizaeunice@hotmail.com
}

1 Universidade Federal de Minas Gerais, Belo Horizonte, Brasil. 


\section{Introdução}

A partir da segunda metade do século XX, condições favoráveis à ocorrência de doenças infecciosas vêm sendo gradativamente substituídas por aquelas relacionadas às doenças crônicas não transmissíveis (DCNT) - como o consumo alimentar inadequado, a prática insuficiente de atividade física, o fumo e o consumo abusivo de álcool 1,2,3 - tanto em países de alta renda quanto naqueles de média e baixa renda 4 . Apesar de muitos países de média e baixa renda ainda enfrentarem problemas relacionados com as doenças infecciosas emergentes 5, a Organização Mundial da Saúde (OMS) estimou já no ano de 2012 que somente as DCNT (como as doenças cardiovasculares, o diabetes tipo II, as doenças respiratórias crônicas e os cânceres) representavam mais de $60 \%$ das mortes no mundo 4 .

Frutas e hortaliças são alimentos indispensáveis para a composição de um padrão saudável de alimentação ${ }^{1}$. Esses alimentos contêm baixa densidade energética e diversos elementos essenciais para a saúde, como vitaminas, minerais, fibras e outros compostos bioativos, favorecendo a manutenção da saúde e do peso corporal 1,6,7. O baixo consumo desses alimentos foi identificado como um dos principais fatores de risco dietético na determinação de anos de vida saudável perdidos, sendo responsável por mais de 3 milhões de óbitos anualmente em todo o mundo e quase 70 mil apenas no Brasil 8,9.

A OMS e a FAO (Organização das Nações Unidas para a Alimentação e a Agricultura), assim como o Ministério da Saúde, recomendam há cerca de 20 anos o consumo mínimo de $400 \mathrm{~g}$ de frutas e hortaliças diariamente (equivalente a cinco porções diárias) como comportamento promotor de saúde e fator de proteção para diversas doenças 1,10. Entretanto, no Brasil, segundo dados da Pesquisa Nacional de Saúde (PNS) em 2013 e do mais recente Inquérito Nacional de Alimentação, realizado em 2008-2009, $37,3 \%$ e menos de $10 \%$ da população atingem as recomendações mínimas de consumo de frutas e hortaliças, respectivamente 11,12. Tal cenário assegura posição de destaque para a promoção do consumo de frutas e hortaliças entre as diretrizes de promoção de alimentação saudável no campo da saúde pública 13,14,15, com o Governo Federal assumindo o compromisso de aumentar o percentual de adultos que consomem frutas e hortaliças regularmente em, no mínimo, 17,8\% (de 36,5 para 43\%) entre 2016 e 2019 16. Desde 2006, o monitoramento de fatores de risco e proteção para DCNT na população (incluindo o consumo de frutas e hortaliças) é baseado em inquérito telefônico anual junto aos adultos das capitais de estados e do Distrito Federal conduzido pelo Ministério da Saúde 17. Resultados gerais desse monitoramento são publicados em relatórios específicos, mas não possibilitam a compreensão aprofundada da tendência dos indicadores 18 . O objetivo do presente estudo é analisar a tendência temporal do consumo de frutas e hortaliças entre adultos nas capitais brasileiras e no Distrito Federal no período entre 2008 e 2016.

\section{Métodos}

\section{Amostragem e coleta de dados}

Os dados utilizados para este trabalho foram coletados pelo Sistema de Vigilância de Fatores de Risco e Proteção para Doenças Crônicas por Inquérito Telefônico (VIGITEL) entre os anos de 2008-2016. O VIGITEL é um inquérito de base populacional destinado a investigar fatores de risco e proteção para o desenvolvimento de doenças crônicas não transmissíveis, incluindo o consumo alimentar, excesso de peso, sedentarismo, nível de atividade física, hábito de fumar, consumo de bebidas alcoólicas e prevenção de câncer 18. Esse sistema, desde 2006, entrevista anualmente uma amostra probabilística de adultos (18 anos ou mais) residentes em domicílios com telefone fixo, nas 26 capitais dos estados do país e no Distrito Federal. São realizadas aproximadamente 2 mil entrevistas em cada cidade, a cada ano de estudo, totalizando cerca de 570 mil entrevistas no período entre 2006 e 2016.

Cadastros das principais operadoras de telefonia fixa do país são utilizados no processo de amostragem em que, em cada domicílio selecionado, um dos moradores adultos é sorteado aleatoriamente para a participação no inquérito. As entrevistas são realizadas por empresa especializada, contratada pelo Ministério da Saúde, e os entrevistadores do sistema são treinados e supervisionados continuamente por técnicos do Ministério da Saúde e de universidades parceiras 18. As entrevistas são realizadas por meio de computadores no sistema CATI (Computer Assisted Telephone Interview, https://www. 
surveysystem.com/interviewing-cati.htm), o que permite agilidade para a realização da entrevista. $\mathrm{O}$ questionário usado foi desenvolvido especialmente para o VIGITEL, sendo composto por cerca de 100 questões curtas e objetivas, e com opções pré-definidas de respostas (questões fechadas). Mais detalhes sobre o processo de amostragem e coleta de dados do VIGITEL são fornecidos no relatório anual do sistema 18 .

As entrevistas realizadas pelo VIGITEL são associadas a fatores de ponderação para que sejam representativas do conjunto total da população adulta de cada cidade. É atribuído um peso a cada indivíduo que visa corrigir diferenças na probabilidade de seleção dos entrevistados (devido a diferentes números de adultos e de linhas telefônicas entre os domicílios) e igualar a composição sociodemográfica da população servida por linhas telefônicas domiciliares àquela da população adulta total de cada cidade em cada ano de levantamento do inquérito (denominado peso pós-estratificação) 18.

O consumo de frutas e hortaliças é investigado no VIGITEL por meio de nove questões identificando a frequência (número de dias da semana) e a intensidade diária (quantidade consumida por dia) de consumo de frutas, sucos naturais de frutas, hortaliças e de hortaliças cruas e cozidas isoladamente. Utilizou-se para a investigação da frequência semanal de consumo o seguinte modelo geral de questão: "Em quantos dias da semana o(a) sr(a) costuma comer (nome do grupo de alimentos)?": (1 a 2 dias | 3 a 4 dias $\mid 5$ a 6 dias | Todos os dias (inclusive sábados e domingos) | Quase nunca | Nunca). Já para a investigação da intensidade diária de consumo, para as frutas e sucos de frutas, usou-se a questão: "Num dia comum, quantas vezes o(a) sr(a) come (nome do grupo de alimentos)?": (1 vez $\mid 2$ vezes $\mid 3$ ou mais vezes); e para as hortaliças cruas e cozidas: "Num dia comum, o(a) sr(a) come (nome do grupo de alimentos)": (no almoço | no jantar ou | no almoço e no jantar).

Dentre as demais informações disponíveis no VIGITEL, foram incluídas na análise também as características sociodemográficas dos indivíduos, como sexo, idade (em anos completos), escolaridade (última série completada) e capitais de residência.

\section{Organização e análise dos dados}

Com base nas respostas fornecidas, foram criados indicadores referentes ao consumo regular e recomendado de frutas e hortaliças. Assumiu-se consumo regular nos casos em que frutas (incluindo sucos naturais) e hortaliças (independentemente do tipo) fossem consumidas em cinco dias ou mais da semana, independentemente da quantidade. Já para o indicador de consumo recomendado, assumiu-se o consumo de uma fruta ou de um copo de suco de fruta como equivalente a uma porção, limitando-se a um o número máximo de porções diárias para sucos e a três o número para frutas (já incluindo os sucos). Para as hortaliças, computou-se um número máximo de quatro porções diárias, situação que caracteriza indivíduos que informam o hábito de consumir hortaliças cruas no almoço e no jantar e hortaliças cozidas também no almoço e no jantar. A recomendação para o consumo de frutas e hortaliças foi considerada alcançada quando o indivíduo referia o consumo de ambos os grupos em pelo menos cinco dias da semana e quando a soma das porções consumidas diariamente totalizava pelo menos cinco porções. Devido a modificações sofridas pelo questionário do VIGITEL, informações coletadas nos anos de 2006 e 2007 não são comparáveis àquelas coletadas nos demais anos e, por isto, foram excluídas deste trabalho.

O sexo dos entrevistados foi classificado em dois grupos (masculino e feminino), a idade em seis faixas (18-24, 25-34, 35-44, 45-54, 55-64 e $\geq 65$ anos), a escolaridade em três níveis (0-8, 9-11 e $\geq 12$ anos de estudos), a capital de residência foi abordada tanto individualmente quanto agrupada segundo região geográfica (Norte, Nordeste, Centro-oeste, Sul e Sudeste).

\section{Análise dos dados}

Inicialmente, a população de estudo foi descrita para cada um dos anos por meio de sua distribuição segundo sexo, faixa de idade, nível de escolaridade e região geográfica. Em seguida, estimou-se o percentual anual de consumo regular e recomendado de frutas e hortaliças, tanto para o conjunto completo da população quanto segundo sexo, faixa de idade, nível de escolaridade, capital e região de residência. Modelos de regressão Prais-Winsten foram então utilizados para a identificação de tendências significativas (de aumento ou diminuição) na variação temporal dos indicadores. Esse 
modelo é baseado na análise de regressão linear e objetiva corrigir o efeito da autocorrelação serial, sendo recomendado em estudos de tendência temporal 19. O indicador de interesse (percentual de consumo regular ou recomendado de frutas e hortaliças) em cada ano era assumido como desfecho (variável dependente) e o ano do levantamento como variável explicativa (expresso como variável contínua). Sendo assim, um coeficiente de regressão positivo indica aumento na variação percentual média anual do indicador no período; se negativo, redução, e quando não foi identificada diferença estatisticamente significativa considerou-se estacionária. Valores significativos desse coeficiente $(\mathrm{p}<$ $0,05)$ indicam a presença de variações consistentes e significativas.

O aplicativo Stata versão 13.1 (https://www.stata.com) foi usado para organização e análise dos dados. Os dados do VIGITEL estão disponíveis para acesso e uso público (Departamento de Informática do SUS - DATASUS, http://www2.datasus.gov.br/DATASUS/index.php? area=0208\&id=28248785) e sua coleta foi aprovada pela Comissão Nacional de Ética em Pesquisa para Seres Humanos do Ministério da Saúde (CAAE: 65610017.1.0000.0008).

\section{Resultados}

A população estudada durante os anos de 2008 a 2016 foi composta por 463.817 adultos ( $\geq 18$ anos), em sua maioria mulheres, adultos jovens (com idade inferior a 45 anos), com escolaridade inferior a 11 anos de estudos e residindo nas regiões Sudeste e Nordeste do país. No conjunto do período estudado, enquanto a proporção de indivíduos com idades entre 18 e 24 anos diminuiu de 17,9\% para 14,8\%, aquela de indivíduos com 45 anos ou mais aumentou significativamente. Da mesma forma, houve diminuição da proporção de indivíduos na faixa inferior de escolaridade, 0 a 8 anos de estudos, de $43,7 \%$ para $32,5 \%$ e aumento da proporção de indivíduos com escolaridade superior a 12 anos de estudos de $21,6 \%$ para $31,6 \%$ (Tabela 1 ).

Cerca de um em cada três indivíduos consumia frutas e hortaliças regularmente ( $\geq 5$ dias/semana) (33\%), com aumento no período, chegando a 35,2\% em 2016 (aumento médio de 1,86\%/ano). Tal aumento foi verificado em ambos os sexos, com os homens apresentando maior magnitude do que as mulheres (2,42\% contra $1,36 \%)$. Também foram identificados aumentos para quatro das seis faixas de idade estudadas (variando entre 1,12\% para indivíduos com idades entre 55 e 64 anos e 2,55\% para aqueles de 25-34 anos) e para todos os níveis de escolaridade (mais intenso entre os indivíduos com 0-8 anos de estudos, 1,71\%) (Tabela 2). Tais aumentos se concentraram, especialmente, no período entre 2011 e 2015, com oscilação negativa em todos os estratos sociodemográficos no ano de 2016 (Tabela 2). Aumentos na frequência de consumo regular de frutas e hortaliças foram verificados também em 15 das 27 capitais estudadas, principalmente entre as capitais das regiões Centro-oeste e Norte (3,37\% e 3,28\%, respectivamente) (Tabela 3$)$.

Verificou-se aumento também no percentual de consumo recomendado de frutas e hortaliças ( $\geq 5 \mathrm{x} / \mathrm{dia}$ ) de $20 \%$ para $24,4 \%$ (com aumento médio anual de $3,32 \%$ no período completo estudado). Novamente, observou-se aumento em ambos os sexos (com maior magnitude entre os homens, em média 4\%) para cinco das seis faixas de idades estudadas (com menor incremento - 2,3\% - para indivíduos com idades entre 55 e 64 anos, e maior incremento - 4,7\% - para aqueles de 25-34 anos) e também para todos os níveis de escolaridade (variando entre 2,76\% para indivíduos com 12 anos ou mais de estudos e 2,97\% para aqueles entre 0-8 anos) (Tabela 4). Uma vez mais, observaram-se aumentos concentrados no período entre 2011 e 2015, com oscilação negativa na maioria dos estratos sociodemográficos no ano de 2016 (Tabela 4). Apenas três das 27 capitais não apresentaram aumentos estatisticamente significativos no percentual de consumo recomendado de frutas e hortaliças (Natal, Recife e São Paulo), observando-se maiores incrementos também entre as capitais das regiões Centro -oeste e Norte (5,12\% e 5,02\%, respectivamente) (Tabela 5). 
Distribuição * (\%) da população adulta ( $\geq 18$ anos) das capitais de estados brasileiros e do Distrito Federal, segundo características sociodemográficas. Sistema de Vigilância de Fatores de Risco e Proteção para Doenças Crônicas por Inquérito Telefônico (VIGITEL), 2008-2016.

\begin{tabular}{|c|c|c|c|c|c|c|c|c|c|c|}
\hline Variáveis & 2008 & 2009 & 2010 & 2011 & 2012 & 2013 & 2014 & 2015 & 2016 & $\begin{array}{c}\text { Variação média } \\
\text { anual (\%) ** }\end{array}$ \\
\hline Total & 54.353 & 54.367 & 54.339 & 54.144 & 45.448 & 52.929 & 40.853 & 54.174 & 53.210 & \\
\hline \multicolumn{11}{|l|}{ Sexo } \\
\hline Masculino & 46,1 & 46,1 & 46,1 & 46,1 & 46,1 & 46,1 & 46,1 & 46,0 & 46,0 & $-0,02 * \star *$ \\
\hline Feminino & 53,9 & 53,9 & 53,9 & 53,9 & 53,9 & 53,9 & 53,9 & 54,0 & 54,0 & $0,02 * * *$ \\
\hline \multicolumn{11}{|l|}{ Idade (anos) } \\
\hline $18-24$ & 17,9 & 17,2 & 17,1 & 16,7 & 16,4 & 15,9 & 15,6 & 15,2 & 14,8 & $-2,34 * * *$ \\
\hline $25-34$ & 25,4 & 25,5 & 25,4 & 25,4 & 25,2 & 25,3 & 25,3 & 25,2 & 25,2 & $-0,10 * * *$ \\
\hline $35-44$ & 20,4 & 20,3 & 20,1 & 20,0 & 19,9 & 19,7 & 19,6 & 19,4 & 19,3 & $-0,69 * * *$ \\
\hline $45-54$ & 16,1 & 16,3 & 16,4 & 16,6 & 16,8 & 16,9 & 17,1 & 17,3 & 17,4 & 0,98 *** \\
\hline $55-64$ & 10,4 & 10,7 & 10,9 & 11,1 & 11,4 & 11,6 & 11,8 & 12,1 & 12,3 & $2,11 * * *$ \\
\hline 65 e mais & 9,8 & 10,0 & 10,1 & 10,2 & 10,4 & 10,5 & 10,6 & 10,8 & 10,9 & $1,38 * * *$ \\
\hline \multicolumn{11}{|c|}{ Escolaridade (anos) } \\
\hline $0-8$ & 43,7 & 42,0 & 40,6 & 38,8 & 36,8 & 36,6 & 35,9 & 34,6 & 32,5 & $-3,52 * \star \star$ \\
\hline $9-11$ & 34,7 & 35,8 & 35,8 & 36,7 & 38,5 & 37,5 & 38,1 & 38,1 & 35,9 & 0,70 \\
\hline 12 e mais & 21,6 & 22,2 & 23,5 & 24,5 & 24,7 & 25,9 & 25,9 & 27,3 & 31,6 & $3,96 * * *$ \\
\hline \multicolumn{11}{|c|}{ Conjunto de capitais da região } \\
\hline Norte & 9,4 & 9,4 & 9,8 & 9,8 & 9,9 & 10,0 & 10,0 & 10,1 & 10,2 & $1,00 * * *$ \\
\hline Nordeste & 25,4 & 25,5 & 25,0 & 25,1 & 25,1 & 25,1 & 25,1 & 25,1 & 25,2 & $-0,13$ \\
\hline Centro-oeste & 11,1 & 11,2 & 11,2 & 11,3 & 11,4 & 11,4 & 11,5 & 11,5 & 11,6 & $0,47 * \star *$ \\
\hline Sudeste & 45,6 & 45,4 & 45,8 & 45,6 & 45,5 & 45,4 & 45,1 & 45,1 & 45,0 & $-0,16 * * *$ \\
\hline Sul & 8,5 & 8,5 & 8,2 & 8,2 & 8,1 & 8,1 & 8,1 & 8,1 & 8,1 & $-0,59 * * *$ \\
\hline
\end{tabular}

* Valores ajustados para equiparar a população total estimada de cada capital para cada um dos anos de estudo;

** Correspondente ao coeficiente da regressão Prais-Winsten do valor do indicador sobre o ano de levantamento; $* * * p<0,05$.

\section{Discussão}

Com base na coleta sistemática de dados realizada pelo VIGITEL junto a mais de 460 mil indivíduos, no período entre 2008 e 2016, identificou-se aumento do consumo de frutas e hortaliças nas capitais brasileiras e no Distrito Federal. Observou-se aumento significativo tanto no percentual de consumo regular de frutas e hortaliças - de 33\% para 35,2\% - quanto no de consumo recomendado - de $20 \%$ para $24,4 \%$. Tais aumentos foram verificados em ambos os sexos, na maioria das faixas de idades, níveis de escolaridade e regiões do país. Verificou-se ainda que grande parte do aumento registrado no período aconteceu no intervalo entre 2011 e 2015, com oscilação negativa no percentual de consumo regular e recomendado de frutas e hortaliças em todos os estratos populacionais pesquisados no ano de 2016.

Informações indicando o baixo percentual de consumo recomendado de frutas e hortaliças pela população brasileira já estão disponíveis desde o início dos anos 2000 20. Segundo os resultados da Pesquisa Mundial de Saúde, de 2003, apenas 13\% dos indivíduos atingiam o nível recomendado de consumo de frutas e hortaliças 20 . Ainda que esse dado deva ser comparado com cautela aos valores obtidos no presente trabalho (devido a diferenças metodológicas entre os inquéritos), a grande diferença entre os percentuais observados é um indicativo de que o consumo de frutas e hortaliças esteja em ascensão por período superior ao investigado neste estudo (período para o qual estão disponíveis dados do VIGITEL). Tal conclusão pode inclusive ser validada baseando-se em dados de coleta domiciliar realizada pela PNS em 2013, indicando que pouco mais de um terço $(37,3 \%)$ da população atendeu à 
Tabela 2

Percentual * da população adulta ( $\geq 18$ anos) que consome frutas e hortaliças em cinco ou mais dias da semana nas capitais dos estados brasileiros e no Distrito Federal, segundo características sociodemográficas. Sistema de Vigilância de Fatores de Risco e Proteção para Doenças Crônicas por Inquérito Telefônico (VIGITEL), 2008-2016.

\begin{tabular}{|c|c|c|c|c|c|c|c|c|c|c|}
\hline Variáveis & 2008 & 2009 & 2010 & 2011 & 2012 & 2013 & 2014 & 2015 & 2016 & $\begin{array}{c}\text { Variação média } \\
\text { anual (\%) ** }\end{array}$ \\
\hline \multicolumn{11}{|l|}{ Sexo } \\
\hline Masculino & 26,4 & 25,7 & 26,0 & 27,5 & 26,9 & 29,6 & 29,4 & 31,3 & 28,8 & $2,42 * \star \star *$ \\
\hline Feminino & 38,6 & 37,8 & 37,2 & 39,0 & 40,1 & 41,5 & 42,5 & 43,1 & 40,7 & $1,36 * \star \star$ \\
\hline \multicolumn{11}{|l|}{ Idade (anos) } \\
\hline $18-24$ & 24,6 & 24,1 & 26,6 & 24,6 & 24,8 & 27,1 & 27,5 & 29,3 & 27,4 & $2,04 * \star \star *$ \\
\hline $25-34$ & 29,6 & 27,1 & 27,5 & 28,4 & 29,4 & 30,6 & 33,9 & 35,3 & 31,7 & $2,55 * * *$ \\
\hline $35-44$ & 31,7 & 33,0 & 30,0 & 32,9 & 33,5 & 34,5 & 33,9 & 35,7 & 33,4 & $1,38 * \star \star$ \\
\hline $45-54$ & 37,0 & 35,1 & 35,5 & 36,5 & 37,7 & 41,0 & 38,7 & 39,2 & 38,1 & 1,14 \\
\hline $55-64$ & 40,7 & 41,1 & 40,4 & 42,9 & 42,1 & 44,6 & 44,6 & 44,6 & 42,2 & $1,12 * \star \star$ \\
\hline 65 e mais & 45,3 & 43,3 & 42,1 & 48,8 & 46,2 & 47,8 & 47,6 & 48,1 & 44,7 & 0,97 \\
\hline \multicolumn{11}{|c|}{ Escolaridade (anos) } \\
\hline $0-8$ & 29,5 & 28,6 & 27,1 & 30,7 & 29,7 & 32,1 & 32,4 & 33,0 & 29,9 & $1,71 * \star \star *$ \\
\hline $9-11$ & 31,0 & 29,6 & 30,4 & 30,5 & 31,2 & 33,4 & 33,4 & 33,8 & 32,0 & $1,29 * \star \star$ \\
\hline 12 e mais & 43,2 & 43,1 & 43,0 & 43,3 & 45,0 & 45,3 & 46,5 & 48,9 & 44,3 & $1,48 * \star *$ \\
\hline Total & 33,0 & 32,2 & 32,0 & 33,7 & 34,0 & 36,0 & 36,5 & 37,6 & 35,2 & $1,86 * \star \star$ \\
\hline
\end{tabular}

* Valores ajustados para equiparar a população total estimada de cada capital para cada um dos anos de estudo;

** Correspondente ao coeficiente da regressão Prais-Winsten do valor do indicador sobre o ano de levantamento; $\star \star * ~ p<0,05$.

recomendação proposta pela OMS para o consumo de frutas e hortaliças 21. Além disso, dados sobre a aquisição de alimentos para consumo domiciliar no Brasil, obtidos pela Pesquisa de Orçamentos Familiares do Instituto Brasileiro de Geografia e Estatística (POF-IBGE), também validam essa conclusão, apontando aumento na participação de frutas e hortaliças no total de alimentos adquiridos de 2,6\% em 2003 para 2,9\% em 200922.

Por mais tímidos que possam parecer os aumentos observados no consumo de frutas e hortaliças no país, deve-se considerar que o percentual de consumo destes alimentos é semelhante ou supera os valores observados em países de renda alta e média alta 23,24,25,26. Dados do Behavior Risk Factor Surveillance System (BRFSS), também coletados por entrevista telefônica e com questionário semelhante ao usado no VIGITEL, mostram que nos Estados Unidos o consumo recomendado de frutas e hortaliças se manteve estável num período de 10 anos (entre 1994 e 2005) 23, variando entre $24,6 \%$ e $25 \%$, em patamar semelhante ao observado atualmente nas capitais brasileiras. Poucos países sul-americanos contam com inquéritos regulares contendo informações sobre o consumo alimentar que possibilitem uma comparação com a realidade mais próxima do Brasil. No Chile, em sentido coincidente, dados da Encuesta Nacional de Salud (ENS), coletados por meio de entrevista domiciliar utilizando um questionário de frequência alimentar semiquantitativo, entre 2010 e 2017 mostram estabilidade do percentual de consumo em cerca de $15 \%$, valor inferior ao observado neste estudo 26,27.

Diferenças na variação temporal do consumo de frutas e hortaliças entre os sexos, faixas de idades e níveis de escolaridade devem também ser consideradas. Um achado importante deste trabalho expõe que grande parte dos estratos populacionais apresentou elevação dos indicadores de consumo e que a variação observada no período estudado foi capaz de reduzir as diferenças encontradas no ano de 2008. Dessa forma, maiores níveis de consumo foram observados durante todo o período entre as mulheres, indivíduos nas faixas superiores de idades e maior nível de escolaridade. Entretanto, uma vez que as maiores variações anuais no percentual de consumo recomendado de frutas e hortaliças foram registradas entre os homens ( $4 \%$ vs. 3,05\% para as mulheres), entre indivíduos nas menores 
Tabela 3

Percentual * de adultos ( $\geq 18$ anos) que consomem frutas e hortaliças em cinco ou mais dias da semana, segundo as capitais dos estados brasileiros e o Distrito Federal. Sistema de Vigilância de Fatores de Risco e Proteção para Doenças Crônicas por Inquérito Telefônico (VIGITEL), $2008-2016$.

\begin{tabular}{|c|c|c|c|c|c|c|c|c|c|c|}
\hline Capitais & 2008 & 2009 & 2010 & 2011 & 2012 & 2013 & 2014 & 2015 & 2016 & $\begin{array}{c}\text { Variação média } \\
\text { anual (\%) ** }\end{array}$ \\
\hline Aracaju & 33,1 & 35,1 & 32,6 & 34,0 & 33,4 & 36,0 & 37,5 & 39,7 & 39,2 & 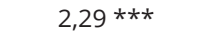 \\
\hline Belém & 21,0 & 19,5 & 20,2 & 24,0 & 24,7 & 27,1 & 26,9 & 24,0 & 24,9 & 2,78 \\
\hline Belo Horizonte & 40,3 & 40,3 & 40,4 & 40,8 & 42,9 & 45,1 & 47,7 & 45,3 & 45,4 & $1,95 * * *$ \\
\hline Boa Vista & 23,1 & 24,5 & 24,6 & 29,0 & 26,2 & 33,7 & 31,2 & 36,9 & 27,3 & $5,42 * \star \star$ \\
\hline Campo Grande & 32,2 & 31,9 & 30,6 & 33,3 & 35,2 & 39,4 & 38,4 & 36,2 & 36,0 & 1,96 \\
\hline Cuiabá & 28,8 & 29,4 & 27,1 & 31,5 & 34,5 & 33,7 & 32,3 & 38,8 & 31,2 & $3,26 * * *$ \\
\hline Curitiba & 38,9 & 39,6 & 37,8 & 40,7 & 42,7 & 43,9 & 47,6 & 44,4 & 44,3 & $2,25 * \star \star$ \\
\hline Florianópolis & 43,5 & 45,6 & 42,5 & 42,1 & 46,0 & 47,5 & 50,1 & 48,1 & 44,4 & 0,95 \\
\hline Fortaleza & 29,2 & 25,6 & 26,7 & 27,8 & 27,9 & 32,0 & 30,7 & 29,6 & 28,8 & 1,25 \\
\hline Goiânia & 33,8 & 33,1 & 34,2 & 35,8 & 38,8 & 42,2 & 36,8 & 48,5 & 42,1 & $4,43 * \star *$ \\
\hline João Pessoa & 38,8 & 37,4 & 35,9 & 39,4 & 39,1 & 38,6 & 41,1 & 37,6 & 41,8 & $0,94 * \star \star$ \\
\hline Macapá & 22,9 & 21,4 & 19,0 & 22,2 & 23,4 & 27,1 & 28,0 & 28,7 & 26,0 & 3,30 \\
\hline Maceió & 31,1 & 31,8 & 29,8 & 31,4 & 31,6 & 33,9 & 38,8 & 30,9 & 32,1 & 1,14 \\
\hline Manaus & 24,9 & 24,1 & 22,3 & 24,9 & 25,8 & 29,1 & 28,6 & 32,0 & 28,0 & $3,58 * \star \star$ \\
\hline Natal & 36,9 & 35,9 & 34,4 & 39,1 & 36,5 & 42,1 & 40,2 & 39,7 & 37,2 & 1,37 \\
\hline Palmas & 30,6 & 27,3 & 30,9 & 36,0 & 33,5 & 41,3 & 38,9 & 40,4 & 34,3 & $3,97 * \star \star$ \\
\hline Porto Alegre & 40,4 & 39,3 & 37,3 & 41,5 & 42,0 & 44,1 & 45,4 & 45,1 & 42,0 & 1,51 \\
\hline Porto Velho & 26,3 & 23,6 & 23,6 & 25,7 & 25,9 & 30,6 & 31,3 & 30,2 & 28,6 & 2,57 \\
\hline Recife & 38,4 & 34,5 & 35,7 & 33,7 & 35,8 & 34,8 & 34,4 & 36,8 & 34,9 & $-0,24$ \\
\hline Rio Branco & 21,0 & 22,0 & 22,4 & 23,8 & 26,7 & 26,9 & 30,6 & 30,5 & 24,9 & $4,05 * \star \star$ \\
\hline Rio de Janeiro & 31,1 & 32,1 & 30,7 & 33,1 & 32,5 & 33,1 & 36,8 & 38,5 & 33,3 & $2,27 \star \star \star$ \\
\hline Salvador & 29,1 & 25,4 & 24,6 & 26,5 & 27,3 & 30,7 & 29,7 & 28,9 & 29,8 & 1,50 \\
\hline São Luís & 24,5 & 22,2 & 24,7 & 26,2 & 24,0 & 26,4 & 29,6 & 27,4 & 26,2 & $2,30 * \star \star$ \\
\hline São Paulo & 34,6 & 32,4 & 33,6 & 35,4 & 35,0 & 36,6 & 35,5 & 36,5 & 33,8 & 0,83 \\
\hline Teresina & 25,2 & 26,3 & 25,4 & 30,0 & 27,1 & 32,2 & 29,1 & 33,2 & 31,0 & $3,35 * \star \star$ \\
\hline Vitória & 37,7 & 39,6 & 35,4 & 39,6 & 41,2 & 43,8 & 39,5 & 44,5 & 42,4 & $2,03 * \star \star$ \\
\hline Distrito Federal & 36,8 & 42,0 & 43,5 & 38,5 & 40,0 & 40,0 & 41,6 & 53,9 & 49,8 & $3,37 * \star \star$ \\
\hline \multicolumn{11}{|c|}{ Conjunto de capitais da região } \\
\hline Norte & 23,5 & 22,4 & 22,1 & 25,2 & 25,8 & 29,2 & 29,1 & 29,9 & 27,1 & $3,28 * \star \star$ \\
\hline Nordeste & 31,2 & 28,8 & 28,8 & 30,4 & 30,3 & 33,1 & 33,1 & 32,3 & 32,0 & 1,24 \\
\hline Centro-oeste & 34,5 & 36,9 & 37,5 & 36,3 & 38,4 & 39,8 & 38,9 & 48,3 & 43,9 & $3,37 * \star \star$ \\
\hline Sudeste & 34,3 & 33,5 & 33,5 & 35,4 & 35,3 & 36,7 & 37,4 & 38,3 & 35,2 & $1,54 * \star \star$ \\
\hline Sul & 40,0 & 40,2 & 38,2 & 41,2 & 42,8 & 44,4 & 47,1 & 45,1 & 43,4 & $1,68 * \star *$ \\
\hline
\end{tabular}

* Valores ajustados para equiparar a população total estimada de cada capital para cada um dos anos de estudo; ** Correspondente ao coeficiente da regressão Prais-Wisten do valor do indicador sobre o ano de levantamento; $* \star * \mathrm{p}<0,05$.

faixas de idades (3,97\% para a faixa entre 18 e 24 anos e 4,7\% para a faixa entre 25 e 34 , superiores a todas as demais faixas) e menor nível de escolaridade (2,97\% para indivíduos com 0-8 anos vs. 2,8\% e $2,76 \%$ para indivíduos com escolaridade superior a 9 anos de estudos), é seguro afirmar que a variação observada no período diminuiu as diferenças relacionadas a esses estratos populacionais. Trata-se de um achado relevante, uma vez que as iniquidades em saúde são importantes preditores da morbidade e mortalidade para DCNT 5,28.

Apesar de o presente estudo não se destinar a investigar as causas da evolução positiva da prevalência de consumo de frutas e hortaliças, acredita-se que isto aconteça, ao menos em parte, ao cenário mais favorável para o consumo no país como resultado de ações do Governo Federal no período 
Percentual * da população adulta ( $\geq 18$ anos) que consome cinco ou mais porções diárias de frutas e hortaliças nas capitais dos estados brasileiros e no Distrito Federal, segundo características sociodemográficas. Sistema de Vigilância de Fatores de Risco e Proteção para Doenças Crônicas por Inquérito Telefônico (VIGITEL), 2008-2016.

\begin{tabular}{|c|c|c|c|c|c|c|c|c|c|c|}
\hline Variáveis & 2008 & 2009 & 2010 & 2011 & 2012 & 2013 & 2014 & 2015 & 2016 & $\begin{array}{c}\text { Variação média } \\
\text { anual (\%) ** }\end{array}$ \\
\hline \multicolumn{11}{|l|}{ Sexo } \\
\hline Masculino & 15,8 & 15,8 & 16,0 & 17,5 & 17,6 & 19,3 & 19,3 & 21,0 & 19,4 & $4,00 * * \star$ \\
\hline Feminino & 23,7 & 23,9 & 22,5 & 25,8 & 27,2 & 27,3 & 28,2 & 28,9 & 28,7 & $3,05 * \star \star$ \\
\hline \multicolumn{11}{|l|}{ Idade (anos) } \\
\hline $18-24$ & 15,6 & 15,7 & 16,9 & 17,3 & 17,7 & 18,9 & 19,2 & 21,0 & 20,7 & $3,97 \star \star \star$ \\
\hline $25-34$ & 18,3 & 17,3 & 17,2 & 19,2 & 20,4 & 21,5 & 22,7 & 25,3 & 22,5 & $4,70 * \star \star$ \\
\hline $35-44$ & 19,4 & 21,5 & 18,0 & 21,0 & 22,5 & 22,8 & 23,4 & 24,2 & 23,6 & $2,95 * * *$ \\
\hline $45-54$ & 22,3 & 21,5 & 22,0 & 23,8 & 24,2 & 26,2 & 25,9 & 26,3 & 25,9 & 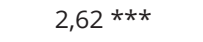 \\
\hline $55-64$ & 23,6 & 25,1 & 25,7 & 27,0 & 28,5 & 29,3 & 28,7 & 28,8 & 28,6 & $2,30 * * \star$ \\
\hline 65 e mais & 26,3 & 25,0 & 22,2 & 29,9 & 28,4 & 26,8 & 27,8 & 27,3 & 28,2 & 1,49 \\
\hline \multicolumn{11}{|c|}{ Escolaridade (anos) } \\
\hline $0-8$ & 16,9 & 16,8 & 15,3 & 18,9 & 18,6 & 19,4 & 20,2 & 20,1 & 19,7 & 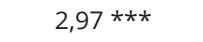 \\
\hline $9-11$ & 19,6 & 19,0 & 19,1 & 20,6 & 21,2 & 23,1 & 22,5 & 23,2 & 23,0 & $2,80 * * \star$ \\
\hline 12 e mais & 27,1 & 28,5 & 27,4 & 28,9 & 31,4 & 30,1 & 31,9 & 34,6 & 30,8 & $2,76 * \star *$ \\
\hline Total & 20,0 & 20,2 & 19,5 & 22,0 & 22,7 & 23,6 & 24,1 & 25,2 & 24,4 & $3,32 * \star \star *$ \\
\hline
\end{tabular}

* Valores ajustados para equiparar a população total estimada de cada capital para cada um dos anos de estudo;

** Correspondente ao coeficiente da regressão Prais-Winsten do valor do indicador sobre o ano de levantamento; $\star \star * \mathrm{p}<0,05$

estudado. A implantação do Plano de Ações Estratégicas para o Enfrentamento das Doenças Crônicas não Transmissíveis (DCNT) no Brasil em 2011 e da Estratégia Intersetorial de Prevenção e Controle da Obesidade em 2014 estabeleceu metas para a ampliação do consumo de frutas e hortaliças, e sugeriram ações intersetoriais a serem adotadas para a ampliação do consumo de alimentos saudáveis, entre os quais frutas e hortaliças 13,29. Significativo esforço do Ministério da Saúde quanto à confecção de materiais técnicos para a educação alimentar e nutricional da população e para a promoção do consumo de frutas e hortaliças por profissionais da saúde, especialmente na Atenção Básica à Saúde, foi registrado no período. Dentre esses se destaca o Guia Alimentar para a População Brasileira (2014) 15, os livros Alimentos Regionais Brasileiros (2015) 30 e Na Cozinha com as Frutas, Legumes e Verduras (2016) 31. Tais publicações não apenas ressaltam a importância do consumo de frutas e hortaliças para a saúde como também apresentam formas para fazê-lo de modo acessível e prazeroso 15,30,31.

O consumo de frutas e hortaliças surge também com destaque no Plano Nacional de Segurança Alimentar e Nutricional (PLANSAN 2016-2019) 16, ressaltando, especialmente, a necessidade de qualificação da mensuração do volume, origem, preços e formatos de produtos comercializados pelas cadeias produtivas de frutas e hortaliças do país e o consumo destes produtos por escolares, povos e comunidades tradicionais. Ainda que não se tenha registros de grandes ações intersetoriais para a promoção do consumo de frutas e hortaliças no país, o percentual de consumo recomendado de frutas e hortaliças almejado para o ano de 2022 (24,3\%) no Plano de Ações Estratégicas para o Enfrentamento das Doenças Crônicas não Transmissíveis no Brasil foi atingido já no ano de 2015, sem que isto tenha sido capaz de interferir na tendência de aumento observada para o percentual de excesso de peso e obesidade na população 15,32 . De fato, considerando os benefícios para a saúde atribuídos ao consumo de frutas e hortaliças, o alto percentual de indivíduos com consumo insuficiente destes alimentos ainda é decepcionante. Cerca de dois a cada três indivíduos (64,8\%) não consomem frutas e hortaliças diariamente. Ainda, um em cada três indivíduos com consumo diário não atinge as recomendações por consumir frutas e hortaliças em quantidade insuficiente. Em função desse cenário, no ano de 2016 a Câmara Interministerial de Segurança Alimentar e Nutricional (Caisan), da qual faz 
Tabela 5

Percentual * de adultos ( $\geq 18$ anos) que consomem cinco ou mais porções diárias de frutas e hortaliças, segundo as capitais dos estados brasileiros e o Distrito Federal. Sistema de Vigilância de Fatores de Risco e Proteção para Doenças Crônicas por Inquérito Telefônico (VIGITEL), $2008-2016$.

\begin{tabular}{|c|c|c|c|c|c|c|c|c|c|c|}
\hline Capitais & 2008 & 2009 & 2010 & 2011 & 2012 & 2013 & 2014 & 2015 & 2016 & $\begin{array}{c}\text { Variação média } \\
\text { anual }(\%) * *\end{array}$ \\
\hline Aracaju & 18,7 & 19,4 & 18,4 & 18,1 & 18,9 & 21,4 & 24,0 & 24,0 & 25,7 & $4,31 * * \star$ \\
\hline Belém & 12,7 & 10,8 & 11,7 & 15,8 & 16,5 & 16,6 & 15,4 & 16,8 & 17,1 & 4,75 *** \\
\hline Belo Horizonte & 23,0 & 24,5 & 25,8 & 26,9 & 29,1 & 29,6 & 31,7 & 30,4 & 31,1 & $3,81 * \star \star$ \\
\hline Boa Vista & 14,0 & 15,3 & 13,2 & 19,6 & 16,7 & 20,5 & 20,8 & 23,1 & 18,8 & $6,29 * * *$ \\
\hline Campo Grande & 18,6 & 19,3 & 19,2 & 20,6 & 23,1 & 24,5 & 26,3 & 24,1 & 23,5 & $3,36 * * *$ \\
\hline Cuiabá & 17,8 & 18,1 & 16,7 & 20,2 & 22,5 & 22,1 & 19,7 & 26,0 & 21,5 & $4,27 * \star \star$ \\
\hline Curitiba & 22,1 & 24,1 & 21,9 & 24,2 & 27,2 & 27,1 & 30,2 & 29,6 & 28,7 & 4,04 *** \\
\hline Florianópolis & 24,9 & 27,4 & 25,9 & 26,9 & 30,3 & 29,8 & 34,7 & 33,4 & 29,3 & $3,34 * * *$ \\
\hline Fortaleza & 14,3 & 13,3 & 13,3 & 16,5 & 16,3 & 18,8 & 18,3 & 17,0 & 18,1 & 3,91 *** \\
\hline Goiânia & 20,9 & 20,9 & 21,7 & 23,5 & 25,8 & 27,6 & 25,0 & 31,6 & 29,1 & $5,34 * * *$ \\
\hline João Pessoa & 20,9 & 20,2 & 19,4 & 20,7 & 21,4 & 22,0 & 23,7 & 21,9 & 24,5 & $2,31 * * \star$ \\
\hline Macapá & 13,8 & 13,4 & 11,4 & 14,6 & 16,3 & 18,0 & 18,9 & 19,8 & 18,2 & 5,35 *** \\
\hline Maceió & 15,9 & 17,1 & 14,0 & 19,1 & 18,9 & 18,3 & 22,4 & 15,6 & 20,9 & $2,72 * \star \star$ \\
\hline Manaus & 15,5 & 15,2 & 14,9 & 15,8 & 16,8 & 18,0 & 19,3 & 21,4 & 19,1 & 4,19 *** \\
\hline Natal & 19,7 & 18,2 & 18,7 & 21,5 & 22,8 & 23,9 & 23,4 & 23,1 & 21,0 & 1,50 \\
\hline Palmas & 18,6 & 16,9 & 19,1 & 24,1 & 23,5 & 26,1 & 26,9 & 29,1 & 24,3 & $6,04 * * *$ \\
\hline Porto Alegre & 23,5 & 22,8 & 21,1 & 25,2 & 27,8 & 28,4 & 28,6 & 29,0 & 28,2 & 3,22 *** \\
\hline Porto Velho & 14,6 & 12,4 & 14,6 & 15,8 & 16,2 & 19,1 & 21,3 & 18,6 & 19,3 & $5,33 * * *$ \\
\hline Recife & 19,8 & 19,4 & 18,4 & 18,7 & 19,3 & 19,5 & 19,1 & 20,4 & 22,2 & 1,40 \\
\hline Rio Branco & 12,3 & 11,5 & 12,8 & 13,9 & 16,0 & 15,7 & 17,1 & 16,9 & 15,8 & $4,39 * * \star$ \\
\hline Rio de Janeiro & 20,0 & 20,6 & 18,6 & 21,5 & 21,6 & 22,2 & 24,7 & 27,8 & 23,4 & 3,98 *** \\
\hline Salvador & 16,5 & 16,2 & 14,0 & 16,7 & 17,1 & 18,6 & 18,6 & 18,6 & 20,3 & $3,20 * * *$ \\
\hline São Luís & 16,0 & 15,0 & 17,3 & 17,9 & 16,9 & 17,7 & 20,4 & 19,5 & 20,1 & $3,49 * * *$ \\
\hline São Paulo & 23,3 & 22,3 & 22,2 & 25,4 & 25,5 & 26,1 & 25,3 & 25,1 & 25,3 & 1,47 \\
\hline Teresina & 13,5 & 17,0 & 15,9 & 18,7 & 17,1 & 19,0 & 19,0 & 22,1 & 20,5 & $4,51 * \star \star$ \\
\hline Vitória & 22,5 & 25,1 & 22,0 & 25,6 & 27,8 & 28,0 & 26,2 & 31,1 & 29,0 & 3,55 *** \\
\hline Distrito Federal & 23,1 & 27,1 & 27,0 & 26,5 & 28,3 & 29,7 & 29,3 & 39,5 & 35,5 & $5,33 * * *$ \\
\hline \multicolumn{11}{|c|}{ Conjunto de capitais da região } \\
\hline Norte & 14,2 & 13,3 & 13,6 & 16,2 & 16,9 & 18,1 & 18,6 & 19,9 & 18,5 & 5,02 *** \\
\hline Nordeste & 16,8 & 16,6 & 15,8 & 18,0 & 18,1 & 19,4 & 20,0 & 19,4 & 20,7 & $3,15 * * \star$ \\
\hline Centro-oeste & 21,3 & 23,4 & 23,4 & 24,2 & 26,3 & 27,6 & 26,8 & 33,8 & 30,6 & $5,12 * * *$ \\
\hline Sudeste & 22,2 & 22,1 & 21,5 & 24,3 & 24,8 & 25,3 & 25,9 & 26,7 & 25,5 & $2,60 * \star \star$ \\
\hline Sul & 23,0 & 24,0 & 22,1 & 24,9 & 27,8 & 27,9 & 30,2 & 29,9 & 28,6 & $3,63 * * *$ \\
\hline
\end{tabular}

* Valores ajustados para equiparar a população total estimada de cada capital para cada um dos anos de estudo;

** Correspondente ao coeficiente da regressão Prais-Winsten do valor do indicador sobre o ano de levantamento;

$* \star * \mathrm{p}<0,05$.

parte o Ministério da Saúde, estabeleceu nova meta para o consumo de frutas e hortaliças, de aumento de $17,8 \%$ no percentual de consumo regular até o ano de 201916.

Apesar desses avanços, sabe-se que intervenções focadas em pequenos grupos populacionais podem aumentar o consumo de frutas e hortaliças em curto prazo no nível local 33,34,35. Entretanto, pouco se sabe sobre a eficácia a longo prazo dessas intervenções (uma vez que o acompanhamento dos indivíduos tende a cessar após o término da intervenção ou a se manter por um curto período, geralmente de um ano) ou mesmo sobre a real efetividade de intervenções intersetoriais para o aumento do consumo desses alimentos no conjunto da população do país. Sendo assim, fatores econômicos, como no caso da renda familiar e o preço (potenciais fatores determinantes do consumo de frutas e 
hortaliças) 36, possivelmente contribuíram com o aumento do consumo entre o período de $2011 \mathrm{e}$ 2015, em vista do crescimento econômico vivenciado no Brasil neste período. Ademais, explicariam também o decréscimo no ano de 2016 pelo aumento do nível de preços dos alimentos e a diminuição da renda em razão da crise econômica no país. Nesse sentido, eventualmente o alcance de níveis de consumo superiores aos atuais envolva a adoção de ações que consideram os determinantes do seu baixo consumo. Assim, estratégias de regulamentação do mercado que oportunizem a produção e distribuição de frutas e hortaliças, como o Programa de Aquisição de Alimentos 37 e a inserção de feiras-livres devem ser privilegiadas 38,39 , uma vez que este tipo de ação poderia solucionar questões como a disparidade do acesso físico e financeiro a estes alimentos 36 .

Contudo, as limitações deste estudo devem ser indicadas. O VIGITEL utiliza entrevistas telefônicas que se propõem a medir o consumo de frutas e hortaliças na população baseando-se na condição referida pelos entrevistados, mais susceptíveis a imprecisões do que aquelas diretamente aferidas 40 . Além disso, o consumo de frutas e hortaliças não pôde ser precisamente aferido em gramas ou porções, baseando-se em informações sobre a frequência semanal e a intensidade diária de consumo (número de vezes num dia habitual para o caso das frutas e número de episódios - almoço, jantar ou almoço e jantar - no caso das hortaliças). Mesmo assim, acredita-se que tais limitações não tenham impactado decisivamente nos resultados apresentados. Informações autorreferidas são frequentemente usadas em grandes inquéritos sobre condições de saúde e estilo de vida (por sua praticidade e menor custo) e técnicas semelhantes às empregadas no VIGITEL são adotadas por outros inquéritos de saúde realizados por entrevista telefônica (como o BRFSS/CDC 41) ou destinadas à investigação de extenso número de fatores de risco (como o STEPS/OMS 42). Para mais, a boa validade e reprodutibilidade dos indicadores de consumo de frutas e hortaliças disponíveis no VIGITEL vem sendo constatada em todas as investigações realizadas até o momento 43,44 . Por último, a restrição da amostra do VIGITEL aos indivíduos que possuem telefone fixo e a realização de número semelhante de entrevistas nas capitais investigadas (independentemente do número de habitantes) pode também originar questionamentos quanto à validade dos dados, especialmente quando capitais de portes distintos são combinadas para a obtenção de uma estimativa (por exemplo, no caso em que as capitais de uma mesma região são combinadas). No entanto, o VIGITEL disponibiliza um fator de ponderação, objetivando, entre outros, igualar a composição sociodemográfica estimada para a população de adultos com telefone em cada capital à composição sociodemográfica da população adulta total da mesma capital. A utilização desse fator (como feito neste trabalho) minimiza a ocorrência de vieses relacionados à cobertura desigual da telefonia fixa entre as cidades investigadas e permite a obtenção de valores para agrupados das capitais, levando em consideração seus diferentes portes 18 .

Por fim, em vista da série histórica ainda limitada no VIGITEL (nove anos de estudos), variações temporais que não tenham sido uniformes ao longo do período (aumento seguido de declínio ou declínio seguido de aumento) tendem a não ser detectadas pela técnica analítica utilizada. Essas tendências apenas poderão ser estudadas de forma acurada quando houver maior acúmulo de dados, o que dependerá da continuidade do sistema.

\section{Conclusão}

A despeito do aumento do percentual de consumo de frutas e hortaliças tanto regular quanto recomendado nas capitais dos estados do país e no Distrito Federal, um percentual relevante da população não atingiu as recomendações mínimas de consumo destes alimentos, evidenciando a necessidade de ações de promoção e incentivo de seu consumo para a população em geral. 


\section{Colaboradores}

L. E. S. Silva e R. M. Claro contribuíram na concepção e delineamento do estudo, análise e interpretação dos dados, redação e revisão do artigo.

\section{Informações adicionais}

ORCID: Luiza Eunice Sá da Silva (0000-00031320-4937); Rafael Moreira Claro (0000-00019690-575X).

\section{Agradecimentos}

Os autores agradecem à Coordenação de Aperfeiçoamento de Pessoal de Nível Superior (Capes) pelo financiamento de bolsa de estudo. Este trabalho contou com o apoio da Capes) (Código de Financiamento 001), do Conselho Nacional de Desenvolvimento Científico e Tecnológico (CNPq, números de concessão 309293/2016-2 e 407331/2016-6) e da Fundação de Amparo à Pesquisa de Minas Gerais (FAPEMIG, números de concessão APQ-02329-15 - 01/2015 e 535 PPM-00325-17 - 02/2017)

\section{Referências}

1. World Health Organization; Food and Agriculture Organization of the United Nations. Diet, nutrition and the prevention of chronic diseases. Report of a Joint WHO/FAO Expert Consultation. Geneva: World Health Organization; 2003. (WHO Technical Report Series, 916).

2. Ezzati M, Lopez AD, Rodgers A, Vander Hoorn S, Murray CJ; Comparative Risk Assessment Collaborating Group. Selected major risk factors and global and regional burden of disease. Lancet 2002; 360:1347-60.

3. Malta DC, Felisbino-Mendes MS, Machado IE, Passos VMA, Abreu DMX, Ishitani LH, et al. Fatores de risco relacionados à carga global de doença do Brasil e Unidades Federadas, 2015. Rev Bras Epidemiol 2017; 20 Suppl 1:217-32.

4. World Health Organization. Global status report on noncommunicable diseases 2014. Geneva: World Health Organization; 2014.

5. Pan American Health Organization. Health in the Americas+, 2017 Edition. Summary: Regional Outlook and Country Profiles. Washington DC: Pan American Health Organization; 2017. (Scientific and Technical Publication, 642).

6. Lock K, Pomerleau J, Causer L, Altmann DR, McKee M. The global burden of disease attributable to low consumption of fruit and vegetables: implications for the global strategy on diet. Bull World Health Organ 2005; 83:100-8.

7. Rolls BJ, Ello-Martin JA, Tohill BC. What can intervention studies tell us about the relationship between fruit and vegetable consumption and weight management? Nutr Rev 2004; 62:1-17.

8. GBD 2016 Risk Factors Collaborators. Global, regional, and national comparative risk assessment of 84 behavioural, environmental and occupational, and metabolic risks or clusters of risks, 1990-2016: a systematic analysis for the Global Burden of Disease Study 2016. Lancet 2017 ; 390:1345-422.
9. Institute for Health Metrics and Evaluation. Global burden of disease 2016. http://vizhub. healthdata.org/gbd-compare/ (acessado em 12/ Mar/2017).

10. Secretaria de Atenção à Saúde, Ministério da Saúde. Guia alimentar para a população brasileira: promovendo a alimentação saudável. Promovendo a alimentação saudável. Brasília: Ministério da Saúde; 2008. (Série A. Normas e Manuais Técnicos).

11. Instituto Brasileiro de Geografia e Estatística. Pesquisa Nacional de Saúde 2013: percepção do estado de saúde, estilos de vida e doenças crônicas. Rio de Janeiro: Instituto Brasileiro de Geografia e Estatística; 2014.

12. Instituto Brasileiro de Geografia e Estatística. Pesquisa de orçamentos familiares 2008-2009: análise do consumo alimentar pessoal no Brasil. Rio de Janeiro: Instituto Brasileiro de Geografia e Estatística; 2011.

13. Departamento de Análise de Situação de Saúde, Secretaria de Vigilância em Saúde, Ministério da Saúde. Plano de ações estratégicas para o enfrentamento das Doenças Crônicas não Transmissíveis (DCNT) no Brasil 2011-2022. Brasília: Ministério da Saúde; 2011. (Série B. Textos Básicos de Saúde).

14. Departamento de Atenção Básica, Secretaria de Atenção à Saúde, Ministério da Saúde. Política Nacional de Alimentação e Nutrição. Brasília: Ministério da Saúde; 2013.

15. Departamento de Atenção Básica, Secretaria de Atenção à Saúde, Ministério da Saúde. Guia alimentar para a população brasileira. 2a Ed. Brasília: Ministério da Saúde; 2014.

16. Câmara Interministerial de Segurança Alimentar e Nutricional. Plano nacional de segurança alimentar e nutricional (PLANSAN 2016-2019). Brasília: Câmara Interministerial de Segurança Alimentar e Nutricional; 2016.

17. Moura EC, Morais NOL, Malta DC, Moura L, Silva NN, Bernal R, et al. Vigilância de Fatores de Risco para Doenças Crônicas por Inquérito Telefônico nas capitais dos 26 estados brasileiros e no Distrito Federal (2006). Rev Bras Epidemiol 2008; 11 Suppl 1:20-37. 
18. Departamento de Vigilância de Doenças e Agravos não Transmissíveis e Promoção da Saúde, Secretaria de Vigilância em Saúde, Ministério da Saúde. Vigitel Brasil 2016: vigilância de fatores de risco e proteção para doenças crônicas por inquérito telefônico. Estimativas sobre frequência e distribuição sociodemográfica de fatores de risco e proteção para doenças crônicas nas capitais dos 26 estados brasileiros e no Distrito Federal em 2016. Brasília: Ministério da Saúde; 2017.

19. Antunes JLF, Cardoso MRA. Uso da análise de séries temporais em estudos epidemiológicos. Epidemiol Serv Saúde 2015; 24:565-76.

20. Jaime PC, Monteiro CA. Fruit and vegetable intake by Brazilian adults, 2003. Cad Saúde Pública 2005; 21Suppl 1:S19-24.

21. Jaime PC, Stopa SR, Oliveira TP, Vieira ML, Szwarcwald CL, Malta DC. Prevalência e distribuição sociodemográfica de marcadores de alimentação saudável, Pesquisa Nacional de Saúde, Brasil 2013. Epidemiol Serv Saúde 2015; 24:267-76.

22. Martins APB, Levy RB, Claro RM, Moubarac JC, Monteiro CA. Participação crescente de produtos ultraprocessados na dieta brasileira (1987-2009). Rev Saúde Pública 2013; 47:65665.

23. Blanck HM, Gillespie C, Kimmons JE, Seymour JD, Serdula MK. Trends in fruit and vegetable consumption among U.S. men and women, 1994-2005. Prev Chronic Dis 2008; 5:A35.

24. Rehm CD, Peñalvo JL, Afshin A, Mozaffarian D. Dietary intake among US adults, 19992012. JAMA 2016; 315:2542-53.

25. NatCen Social Research. Health Survey for England 2016: adult health trends. London: Health and Social Care Information Centre; 2017.

26. Departamento de Epidemiología, División de Planificación Sanitaria, Subsecretaria de Salud Pública. Tercera Encuesta Nacional de Salud (ENS) 2016-2017: primeiros resultados. Santiago de Chile: Ministerio de Salud; 2017.

27. Ministerio de Salud; Pontificia Universidad Católica de Chile; Universidad Alberto Hurtado. Encuesta Nacional de Salud 2009-2010. Santiago de Chile: Ministerio de Salud; 2010.

28. Enes CC, Nucci LB. Gender and schooling inequalities in risk and protective factors for chronic diseases among Brazilian adults. J Public Health (Oxf) 2018; 40:e211-e8

29. Câmara Interministerial de Segurança Alimentar e Nutricional. Estratégia Intersetorial de Prevenção e Controle da Obesidade: recomendações para estados e municípios. Brasília: Câmara Interministerial de Segurança Alimentar e Nutricional; 2014.

30. Departamento de Atenção Básica, Secretaria de Atenção à Saúde, Ministério da Saúde. Alimentos regionais brasileiros. 2a Ed. Brasília: Ministério da Saúde; 2015.

31. Ministério da Saúde; Universidade Federal de Minas Gerais. Na cozinha com as frutas, legumes e verduras. Brasília: Ministério da Saúde; 2016.
32. Malta DC, Silva Jr. JB. Plano de Ações Estratégicas para o Enfrentamento das Doenças Crônicas Não Transmissíveis no Brasil após três anos de implantação, 2011-2013. Epidemiol Serv Saúde 2014; 23:389-95.

33. Jaime PC, Machado FMS, Westphal MF, Monteiro CA. Educação nutricional e consumo de frutas e hortaliças: ensaio comunitário controlado. Rev Saúde Pública 2007; 41:154-7.

34. Franco AS, Castro IRR, Wolkoff DB. Impact of the promotion of fruit and vegetables on their consumption in the workplace. Rev Saúde Pública 2013; 47:29-36.

35. Wagner MG, Rhee Y, Honrath K, Salafia EHB, Terbizan D. Nutrition education effective in increasing fruit and vegetable consumption among overweight and obese adults. Appetite 2016; 100:94-101.

36. Claro RM, Monteiro CA. Renda familiar, preço de alimentos e aquisição domiciliar de frutas e hortaliças no Brasil. Rev Saúde Pública 2010; 44:1014-20.

37. Secretaria Especial de Agricultura Familiar e do Desenvolvimento Agrário, Ministério da Agricultura, Pecuária e Abastecimentos. Programa de Aquisição de Alimentos. http:// www.mda.gov.br/sitemda/secretaria/saf-paa/ sobreo-programa (acessado em 12/Mar/2017).

38. Duran AC, Diez-Roux AV, Latorre MRDO, Jaime PC. Neighborhood socioeconomic characteristics and differences in the availability of healthy food stores and restaurants in São Paulo, Brazil. Health Place 2013; 23:39-47.

39. Pessoa MC, Mendes LL, Gomes CS, Martins PA, Velásquez-Meléndez G. Food environment and fruit and vegetable intake in a urban population: a multilevel analysis. BMC Public Health 2015; 15:1012.

40. Szolnokin G, Hoffmann D. Online, face-toface and telephone surveys: comparing different sampling methods in wine consumer research. Wine Economics and Policy 2013; 2:57-66.

41. Centers for Disease Control and Prevention. Behavioral Risk Factor Surveillance System BRFSS. https://www.cdc.gov/brfss/about/in dex.htm (acessado em 24/Jan/2017).

42. World Health Organization. Summary: surveillance of risk factors for non-communicable diseases. The WHO STEP wise approach. Geneva: World Health Organization; 2001.

43. Monteiro CA, Moura EC, Jaime PC, Claro RM. Validade de indicadores do consumo de alimentos e bebidas obtidos por inquérito telefônico. Rev Saúde Pública 2008; 42:582-9.

44. Neves ACMD, Gonzaga LAA, Martens IBG, Moura EC. Validação de indicadores do consumo de alimentos e bebidas obtidos por inquérito telefônico em Belém, Pará, Brasil. Cad Saúde Pública 2010; 26:2379-88. 


\section{Abstract}

The study aimed to analyze the time trend in the consumption of fruits and vegetables among adults in Brazilian state capitals and the Federal District from 2008 to 2016. This was a time series study with data from the VIGITEL survey from 2008 to $2016(n=463,817)$. The study analyzed the prevalence of regular consumption ( $\geq 5$ days/week) and recommended consumption ( $\geq 5$ times/day) of fruits and vegetables, for each of the years, for the complete set of the evaluated population and according to sex, age group, schooling, and location. Presence of linear trend (increase or decrease) in the indicators' variation was analyzed by PraisWinsten regression. There was a significant increase $(p<0.05)$ in prevalence of regular consumption (from 33 to $35.2 \%$, an increase of $1.86 \% /$ year) and recommended consumption of fruits and vegetables (from 20 to $24.4 \%$, an increase of 3.32\%/ year). A similar trend was identified in the percentage of the population meeting the recommended consumption in most of the population strata, with the highest increase in men (4\%/year vs. $3.05 \% / y e a r$ for women), young adults (3.97\%/ year in the 18-24-year group vs. 2.30\%/year in the 55-64-year group), those with low schooling $(2.97 \% / y e a r$ for 0 to 8 years of schooling $v s$. $2.76 \% /$ year for 12 years or more), and less developed regions of Brazil (5.02\%/year in the North vs. 2.6\%/year in the Southeast). There was an increase in the consumption of fruits and vegetables, especially among groups with lower levels of consumption at the start of the study period. However, three out of four individuals still consumed fewer fruits and vegetables than recommended.

Fruits; Vegetables; Food Consumption; Chronic Disease

\section{Resumen}

El objetivo de este estudio fue analizar la tendencia temporal del consumo de frutas y hortalizas entre adultos en las capitales brasileñas y Distrito Federal durante el período entre 2008 y 2016. Se trata de un estudio de serie temporal, con datos procedentes de VIGITEL entre 2008 y 2016 ( $n=463.817)$. Se analizó el porcentaje de consumo regular ( $\geq 5$ dias/semana) y recomendado ( $\geq 5 x$ / día) de frutas y hortalizas, en cada uno de los años, para el conjunto completo de la población y según sexo, franja de edad, nivel de escolaridad y lugar de residencia. La presencia de una tendencia lineal (de aumento o disminución) en la variación de los indicadores se analizó mediante la regresión PraisWinsten. Se verificó un aumento significativo $(p<0,05)$ en la prevalencia del consumo regular (de 33 a 35,2\%, un aumento de 1,86\%/año) y del consumo recomendado de frutas y hortalizas (del 20 al 24,4\%, un aumento de 3,32\%/año). Una tendencia semejante se identificó respecto al porcentaje de la población que respondía a la recomendación de consumo en la mayor parte de los estratos poblacionales, con mayor magnitud de aumento entre: hombres (4\%/año vs. 3,05\%/año para las mujeres), adultos jóvenes (3,97\%/año para aquellos con edad entre 18 y 24 años vs. 2,30\%/año para aquellos con 55-64 años), aquellos con menor escolaridad (2,97\%/año para aquellos de 0 a 8 años de estudios vs. 2,76\%/año para aquellos con 12 años o más) y residentes en regiones menos desarrolladas (5,02\%/año en la región norte vs. 2,6\%/año en la región sudeste). Se observó un aumento del consumo de frutas y hortalizas, especialmente, entre los grupos con menor nivel de consumo durante el inicio del periodo estudiado. No obstante, 3 de cada 4 individuos siguen consumiendo menos frutas $y$ hortalizas de lo recomendado.

Frutas; Hortalizas; Consumo de Alimentos; Enfermedad Crónica
Recebido em 07/Fev/2018

Versão final reapresentada em 17/Dez/2018

Aprovado em 24/Jan/2019 